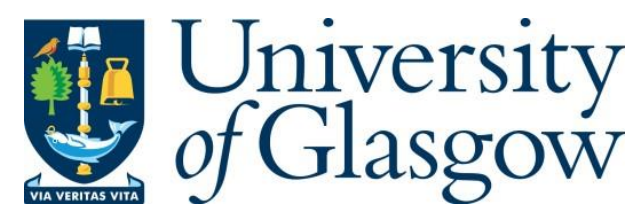

Laban, G. (2021) Perceptions of Anthropomorphism in a Chatbot Dialogue: the Role of Animacy and Intelligence. In: 9th International Conference on HumanAgent Interaction (HAI '21), 09-11 Nov 2021, pp. 305-310. ISBN 9781450386203.

There may be differences between this version and the published version. You are advised to consult the publisher's version if you wish to cite from it.

(C) The Author 2021. This is the author's version of the work. It is posted here for your personal use. Not for redistribution. The definitive Version of Record was published in Proceedings of the 9th International Conference on Human-Agent Interaction (HAI '21), 09-11 Nov 2021, pp. 305-310. ISBN 9781450386203. https://doi.org/10.1145/3472307.3484686.

http://eprints.gla.ac.uk/258806/

Deposited on: 12 November 2021

Enlighten - Research publications by members of the University of Glasgow http://eprints.gla.ac.uk 


\title{
Perceptions of Anthropomorphism in a Chatbot Dialogue: The Role of Animacy and Intelligence
}

\author{
Guy Laban \\ Guy.Laban@Glasgow.ac.uk \\ Institute of Neuroscience and Psychology, University of Glasgow \\ Glasgow, United Kingdom
}

\begin{abstract}
When interacting with embodied agents, users often rely on a variety of cues from the agent's embodiment to form perceptions of animacy and intelligence, including its appearance and behaviour. Due to chatbots' disembodiment, users' perceptions of a chatbot's animacy and intelligence are mostly dependent on the textual properties of the dialogue. The current study aims to investigate the mediating role of perceptions of chatbot's intelligence and animacy on users' perceptions of the chatbot's anthropomorphism. An online experiment was conducted with a chatbot and a web platform. Both systems asked users three basic questions for providing a restaurant recommendation. By communicating the same content via different modalities of communication (i.e., flowing dialogue and traditional web interface); this study compares the differences in these perceptions between chatbots to traditional web platforms. The results of a mediation analysis entail that the chatbot was perceived as more animate than the web platform, and accordingly, it was perceived as more anthropomorphic than the web platform as users' perceptions of animacy fully mediated this effect. Also, there is no evidence for differences in users' perceptions of intelligence between the chatbot and the web platform.
\end{abstract}

\section{CCS CONCEPTS}

- Human-centered computing $\rightarrow$ Empirical studies in HCI; HCI theory, concepts and models; User studies; Text input; Graphical user interfaces; • Applied computing $\rightarrow$ Psychology; Online shopping.

\section{KEYWORDS}

Chatbots, Animacy, Intelligence, Anthropomorphism, Perception, Disembodied Interactions

\section{ACM Reference Format:}

Guy Laban. 2021. Perceptions of Anthropomorphism in a Chatbot Dialogue: The Role of Animacy and Intelligence. In Proceedings of the 9th International Conference on Human-Agent Interaction (HAI '21), November 9-11, 2021, Virtual Event, Japan. ACM, New York, NY, USA, 6 pages. https://doi.org/10. $1145 / 3472307.3484686$

\section{INTRODUCTION}

Anthropomorphism, the attribution of human characteristics to non-human entities [38], has been studied thoroughly in the settings of human-robot interaction (HRI) (e.g., [5][7][13][17]). However, previous studies tend to focus on the role of "human-like" visual and behavioural features when individuals anthropomorphise embodied agents (e.g., social robots and avatars) [26] as these convey humane features clearly and explicitly [50]. Moreover, anthropomorphism in HRI is often situated within clear distinctions of human and machine (e.g., [5][11][37]). Chatbots, also known as conversational agents, are artificially intelligent computer programs that interact with users in a flowing dialogue by using natural language [23], and are often designed to communicate in a humanlike way [45]. When interacting with chatbots online people tend to anthropomorphise these agents [15][29][38] and often perceive, describe, and evaluate these in a human-like manner [2] [31]. Disembodied conversational agents like chatbots utilize different aspects in an interaction to convey a sense of anthropomorphism. These tend to be features of the dialogue's content, modality, the textual and lexical properties of the dialogue and the appearance of it (e.g., [2][19][30][31][46][50]). While previous studies in HRI can clearly state how different features of an embodied agent or an embodied interaction effect the way users anthropomorphise it, there is a literature gap regarding the effect of different disembodied dialogue features on online users anthropomorphic perceptions of disembodied conversational agents (i.e., chatbots).

The current study aims to investigate the mediating role of perceptions of a chatbot's intelligence and animacy, on users' perceptions of the chatbot's anthropomorphism. Animacy is the agent's capability to demonstrate lifelike behavior, whereas intelligence is the agent's ability to acquire and apply knowledge and skills [5]. While animacy is often observed from an individual's appearance and behaviour [4][5][44], intelligence is often observed from an individual's shared content and performance [4][47][48][52][5]. Thus, this study is particularly interested in further understanding how these two contrasting, yet related features mediate anthropomorphic perceptions.

Perceptions of a speaker's animacy and intelligence are established by different social cues within the dialogue. However, when interacting with artificial agents, previous studies reported that these two perceptions are correlated [4]. When interacting with embodied agents, users often rely on a variety of social cues from the agent's embodiment to form perceptions of animacy and intelligence, including its appearance, behaviour, and social role (see [5][9][10][11][28][27][32][33]). Due to chatbots' disembodiment, users' perceptions of a chatbot's animacy and intelligence are mostly dependent on the textual and lexical properties of the 
dialogue, and its content [50]. There is limited knowledge regarding how features that are manifested from text can explain people's anthropomorphic perceptions of chatbots and other disembodied conversational agents that relay mostly on textual dialogue. Hence, this study is aimed at answering the following research question: To what extent users' perceptions of chatbot's dialogue features, animacy and intelligence, mediates users' perceptions of chatbot's anthropomorphism?

Two systems were developed for this study, a chatbot and a web platform. Both systems asked users three basic questions for providing a restaurant recommendation. By communicating the same content via different modalities of communication (i.e., flowing chat dialogue and static traditional web graphic interface); this study compares the differences in these perceptions between chatbots to traditional web platforms. Therefore, this study is aimed at further understanding the role of dialogue and natural language in forming perceptions of intelligence and animacy, and in turn, perceiving the system as more anthropomorphic.

\section{THEORETICAL FRAMEWORK}

\subsection{Stimulating Perceptions of Animacy in a Dialogue}

The media richness theory (MRT) [12] explains that a communication medium's ability to reproduce the information sent through it is driven by its "richness", being its abilities to demonstrate or deliver social cues. Hence, interpersonal communication behaviours would typically perform better when communicated via media with the capacity to convey richer social cues, like gestures and body language [8][12]. However, considering that disembodied information systems (e.g., chatbots and web platforms) are limited from demonstrating a range of behavioural social cues, these are dependent on different textual and lexical properties of the dialogue [50]. Animacy is inherently related to human behaviour [4] [5][44] and is often observed from visual stimuli cues [35][36]. Nevertheless, animacy can also be observed via grammatical and semantic features [20]. By manipulating language, imitating human conversation, and communicating via a dialogue, disembodied conversational agents like chatbots can provide richer interactions [50] as a richer medium [12]. Therefore, it is expected that users will perceive a chatbot to be more animate than a web platform. Hence, the first hypothesis of the study is: H1: Online users will perceive a chatbot as more animate than a web platform.

\subsection{Perceiving Intelligence Objectively and Subjectively}

Intelligence is often observed from the interplay of conventional, formal and objective intelligence, with subjective aspects of intelligence. The first is related to the interaction's content (e.g., the intellectual value of the interaction, logic, state of matters) [47][48][52] and to the platform's responsiveness and competence (i.e., the capacity to perform the required action) [4][5]. Subjective aspects of intelligence are related to the social dimensions and capabilities of the platform, as the capacity to demonstrate and respond to emotions and social information, as well as to understand and react to individual contexts [14][34][40][47][48]. Research into the theory of mind processes posits that people ascribe mental capacities to other entities - human and nonhuman - and then react to and evaluate these based on their moral judgments and values [15][22][53]. While artificial agents do not (yet) offer the same opportunities as humans for social interactions [9], their cognitive architectures and embodied cognition can nonetheless prime people's perceptions of these as socially present (e.g., [2][31][38][43]). Accordingly, it is expected that users will read the chatbot's demonstrated social cues and will perceive it as more intelligent than the web platform. Hence, the second hypothesis of this study is: H2: Online users will perceive a chatbot as more intelligent than a web platform.

\subsection{Anthropomorphizing Information Systems}

Making anthropomorphic inferences during an interaction is an intuitive and mindless task. Nevertheless, it requires cognitive efforts and is more likely to be triggered when certain social cues are present [1][6][28][53]. According to Epley and colleagues [16], people are more likely to anthropomorphise when anthropocentric knowledge is accessible and applicable. Moreover, people often grant a social role to computers during interactions and perceive them as social actors [38][39][42]. It is proposed by the computers are social actors (CASA) paradigm [38][39][42] that humans are mindlessly biased towards a media (e.g., computers, systems, and for the scope of this study also conversational agents) social activity, and when presented with social cues (for the scope of this paper, like animacy and intelligence) people tend to anthropomorphize the system or the agent and respond accordingly. Therefore, it is expected that perceptions of animacy and intelligence will positively mediate users' perceptions of the information system (i.e., the chatbot and the web platform) anthropomorphism. Accordingly, the finally hypothesis for this study is: H3: Online users will perceive a chatbot as more anthropomorphic than a web platform, via the mediating role of (a) perceived animacy and (b) perceived intelligence.

\section{METHODS}

\subsection{Population}

A total of 163 participants were recruited using Amazon Mechanical Turk (MTurk). To minimize the influence of culture and language, the sample was composed of U.S.-based participants who speak English as their first language. Three participants were dropped because of technical issues, resulting in a final sample of 160 participants, with ages ranging between 20 and 65 years old $(M=35.58$, $S D=11.38), 46.9 \%$ identified as females, and with most participants having completed a Bachelor's degree (56.3\%).

\subsection{Design and Stimuli}

The current study used an online experiment with a chatbot and a web platform that were designed for this study. A two (chatbot vs. web platform) between-subjects factors online experiment was conducted. The chatbot for the study was created using the Conversational Agent Research Toolkit [3] as a disembodied conversational agent with a human name ("Emma") that spoke using first-person singular pronouns (e.g., "I am"). Emma communicated with the participants via online chat, manipulating language and dialogue while describing recommendations with nouns and adjectives (e.g., "These restaurants should provide a lavish experience!" 
when describing expensive restaurants). Emma also used greetings (e.g., "Hi") and reacted to users' statements (e.g., "My pleasure!")(see Figure 1).

The web platform utilized buttons, icons, and windows (e.g., a "Go" button to submit values and a pop-up window to show recommendations), to stimulate the impression of a standard website [49]. Recommendations were described with a passive voice and common symbols and icons (e.g., "\$" for describing budget)(see Figure 2).

Both systems contained pre-defined lists of values that corresponded to potential answers of the participants for providing recommendations. For example, pasta is an item in an Italian food list and "not much" is an item in a "small budget" list. Together the system can retrieve potential recommendations that answer to those values (i.e., an Italian restaurant for a small budget).

\subsection{Measurements}

Three scales by Bartneck et al. [5] were applied in the study: Perceived Anthropomorphism $(\alpha=.93, M=3.93, S D=1.76)$, Perceived Intelligence ( $\alpha=.94, M=5.14, S D=1.42)$, and Perceived Animacy $(\alpha=.91, M=4.38, S D=1.63)$.

\subsection{Procedure}

Participants were informed regarding their rights and were asked to provide their informed consent; participants who consented then began the experiment. First, participants answered a set of demographic questions and an attention check. Then, they were randomly assigned to one of the four groups and received corresponding instructions. Using either a chatbot or a web platform, participants answered three open-ended questions-disclosing a favourite cuisine, their budget for a meal, and a preferable location for a restaurant. Accordingly, the system provided a restaurant recommendation. Participants were informed that the manipulation should not take more than three minutes.

After completing the task, participants evaluated the platform in terms of the platform's anthropomorphism, intelligence, and animacy. The order of the questions and scales was randomized. Once participants completed the experiment, they were debriefed about the study and provided with researcher contact information. The study received an ethics review board approval.

\section{RESULTS}

A mediation analysis was conducted using Model 4 of PROCESS Macro 3.2.01 [24][25] to investigate the research hypotheses. The model included the experimental conditions as a dichotomous binary predictor variable, perceived anthropomorphism as the outcome variable, and perceived intelligence and perceived animacy as mediators. Moreover, the model controlled for the confounding influence of the participants' age and identified gender.

\subsection{Direct Effect of the experimental conditions on Perceived Anthropomorphism.}

The results indicate that in step 1 of the mediation model, the chatbot was perceived to be significantly more anthropomorphic than the web platform; $R=.26, F(3,156)=3.60, p=.015$, with the model explaining $7 \%\left(R^{2}=.07\right)$ of the variance in perceived anthropomorphism. The regression of the experimental conditions variable on perceived anthropomorphism was significant, $\beta=.75, t(156)=8.10$, $p=.007,95 \%$ CI $[.21,1.29]$ when cancelling the mediators' effect in the model. Neither gender $(\beta=.35, t(156)=1.28, p=.204,95 \% \mathrm{CI}$ $[-.19, .89])$ nor age $(\beta=-.01, t(156)=-1.01, p=.313,95 \%$ CI $[-.04, .01])$ had a significant effect on perceived anthropomorphism.

\subsection{Direct Effect on Perceived Animacy.}

The model explaining perceived animacy was found to be significant $(R=.23, F(3,156)=2.87, p=.038)$, explaining $5 \%\left(R^{2}=.05\right)$ of the variance in perceived animacy. The chatbot was significantly perceived as more animate compared to the web platforms, $\beta=.67$, $t(156)=2.61, p=.010,95 \%$ CI $[.16,1.17]$. Neither gender $(\beta=.14$, $t(156)=.55, p=.586,95 \%$ CI $[-.36, .64])$ nor age $(\beta=-.01, t(156)=$ $-1.18, p=.238,95 \% \mathrm{CI}[-.04, .01])$ had a significant effect on perceived animacy. Hence, $\mathrm{H} 1$ is supported.

\subsection{Direct Effect on Perceived Intelligence.}

Step 2 of the mediation model showed that the model explaining perceived intelligence was not significant $(R=.15, F(3,156)=1.23$, $p=.338)$, explaining $2 \%\left(R^{2}=.02\right)$ of the variance in perceived intelligence. The chatbot and the web platform were not perceived differently in terms of perceived intelligence, $\beta=.23, t(156)=1.02$, $p=.308,95 \%$ CI $[-.22, .68]$. Neither gender $(\beta=-.17, t(156)=-.74, p$ $=.461,95 \% \mathrm{CI}[-.62, .28])$ nor age $(\beta=-.01, t(156)=-1.45, p=.149$, $95 \% \mathrm{CI}[-.03, .01])$ had a significant effect on perceived intelligence. Therefore, $\mathrm{H} 2$ is not supported.

\subsection{Direct Effect of the mediators on Perceived Anthropomorphism.}

Step 3 of the mediation model showed that the overall model was significant; $R=.84, F(5,154)=72.90, p<=.001)$, with the model explaining $70 \%\left(R^{2}=.70\right)$ of the variance in perceived anthropomorphism. Controlling for the experimental conditions variable, the mediator of perceived animacy $\left(\beta=.84, t(154)=13.48, b^{*}=.78\right.$, $p<.001,95 \% \mathrm{CI}[.72, .96])$ was found to be significant, while the mediator of perceived intelligence, controlling for the experimental conditions variable, was not significant $\left(\beta=.08, t(154)=1.13, b^{*}=\right.$ $.06, p=.261,95 \% \mathrm{CI}[-.06, .22])$. Neither gender $(\beta=.25, t(154)=1.57$, $\left.b^{*}=.07, p=.119,95 \% \mathrm{CI}[-.06, .55]\right)$ nor age $(\beta=.00, t(154)=.02$, $\left.b^{*}=.00, p=.985,95 \% \mathrm{CI}[-.01, .01]\right)$ had a significant effect on perceived anthropomorphism when controlling for the experimental conditions variable.

\subsection{Indirect Effects.}

Step 4 of the analyses revealed that, when controlling for the mediators, the experimental conditions variable was not a significant predictor of perceived anthropomorphism, $\beta=.17, t(154)=1.09, b^{*}$ $=.10, p=.278,95 \% \mathrm{CI}[-.14, .49]$.

Mediation analyses based on 5000 bootstrapped samples using bias-corrected and accelerated 95\% confidence intervals [41] showed that the experimental conditions variable had a significant total effect on the perceived anthropomorphism $(c=.43, S E=.27, p$ $=.007,95 \% \mathrm{CI}[.21,1.29])$, a not significant residual direct effect $\left(c^{\prime}=\right.$ 
$.10, S E=.16, p=.278,95 \% \mathrm{CI}[-.14, .49])$, and a significant indirect effect through perceived animacy $(a b=.56, S E=.21, B C a \mathrm{CI}[.15, .98])$. The indirect effect is significantly different from zero at $p<.05$. Perceived animacy fully mediated the total effect between the experimental conditions variable and perceived anthropomorphism, $a b_{c s}=.32, S E=.12, B C a \mathrm{CI}[.09, .56]$. The results demonstrate that the chatbot was perceived as more animate than the web platform, and accordingly, it was perceived as more anthropomorphic than the web platform. Therefore, H3a is supported whereas H3b is not.

\section{DISCUSSION}

This study was aimed at investigating how users' perceptions of intelligence and animacy differ between a chatbot and a web platform. Moreover, this study was focused on further explaining how perceptions of intelligence and animacy mediate users' anthropomorphic perceptions of the platform. The results of an online experiment show that the chatbot was perceived as more animated than the web platform, and accordingly, it was perceived as more anthropomorphic than the web platform as users' perceptions of animacy fully mediated this effect. In addition to these, there is no evidence for differences in users' perceptions of intelligence between the chatbot and the web platform, and also no effect of perceptions of intelligence on perceptions of anthropomorphism.

These findings are in line with the MRT framework [12] and highlight the value of a communication medium demonstrating and transmitting social cues and gestures. While it is not surprising, and not substantially novel, it is important and meaningful for several reasons. Animacy is inherently related to human behaviour [4][5][44] and is often observed from visual stimuli cues [35][36] Nevertheless, this study's results provide evidence for perceptions of animacy in a textual dialogue. Animacy is rarely discussed in the context of disembodied conversational agents [50] as it is often addressed as an embodied feature to embodied agents (e.g., social robots and avatars) due to their visual appearance [9][10][28][27] The results of this study provides preliminary evidence for perceptions of animacy in disembodied interactions, contribute to the conceptualization of animacy within the context of disembodied interactions as a feature of modality and verbal appearance. Following this key finding, it can be assumed that socially and emotionally oriented communication and behaviour triggers animacy perceptions from textual and lexical properties in a dialogue. Furthermore, the MRT framework [12] was originally concerned with computer-mediated communication (CMC), and accordingly, social cues within the MRT framework are bound to human origins. This key result contributes to the dimensions of the framework, demonstrating the possibility of extending the framework to CMC interactions with artificial agents.

These findings also provide further evidence to the social and cognitive frameworks of anthropomorphism in interactions with artificial agents (e.g., three-factor theory [16], mind perception [15][22][53], CASA [38][39][42]) while differentiating between features of modality and content as accessible and applicable anthropocentric knowledge. Intelligence, as a feature that is often observed in an interaction from the interaction's content [47][48][52] and competence [4][5] is not mediating perceptions of anthropomorphism as would be expected within the field of artificial agents.
However, animacy as a feature of a dialogue's modality [50] express a sense of behaviour in the dialogue [4][20][44] that is of human-origins [10]. This key result suggests that in disembodied interactions, textual and lexical parameters could convey a sense of a "human-like" interaction, and affect the user to perceive a chatbot in an anthropomorphic manner. This is in line with earlier results of Go and Sundar [21] that found that high level of message interactivity, a feature of dialogue modality, compensates for the impersonal nature of a chatbot that is low on anthropomorphic visual cues. Hence, features of modality as animacy, interactivity, and even features that are as broad as the agent's medium of communication (see [51]) can have a substantial impact on how users' perceive the agent beyond the domains of the feature.

Finally, these findings stress the attention users give to conventional and objective intelligence over subjective intelligence [47][48][52] when evaluating information systems. It seems that users pay attention to the system's competency and performance when evaluating the system's intelligence, and are not paying attention to the system' social capabilities. Future studies that aim to evaluate users' perceptions of chatbots' intelligence should extend from Bartneck and colleagues' [5] measures of perceived intelligence, and aim to capture both objective and subjective dimensions of perceived intelligence. When applied to information systems, people pay attention to cues of conventional and objective information like the content of the interaction and the competence and responsiveness of the system. Hence, information systems that might not appear to be humane at all are still valued to be as intelligent if these perform in line with their expectations. Users' relay on the communicated content and the systems' competence to complete the task, and as both systems demonstrated high competence the evaluation of both was similar. This key finding support previous research (e.g., [18][30][31]) that highlights the importance of high quality content (e.g., quality of information, transparency, providing adequate answers) in eliciting positive perceptions.

\section{CONCLUSIONS}

Perceptions of a Chatbot's anthropomorphism are different from perceptions of a web platform by being mediated through perceptions of animacy and not by perceptions of intelligence. These agents are being perceived as more human-like due to their animate way of communicating information rather than due to the intelligent value of the content they communicate or their performance completing the task. Users do not perceive chatbots' intelligence differently from web platforms, and accordingly, it does not appear as a significant source for users' perception of chatbot's anthropomorphism. This study contributes to the conceptualization of animacy within the context of disembodied interaction as a feature of modality and verbal appearance. At the same time, it highlights the universality of intelligence as a feature that is not limited to humans, nor to non-human objects that appear to be human-like. Information systems that might not appear to be humane at all are still valued to be as intelligent based on their competence. In disembodied interactions that are limited in social cues, socially and emotionally oriented communication and behaviour serves as an indicator for human animacy only, and not for intelligence when evaluating information systems. 


\section{REFERENCES}

[1] Gabriella Airenti, Marco Cruciani, and Alessio Plebe. 2019. Editorial: The Cognitive Underpinnings of Anthropomorphism. Frontiers in Psychology 10 (2019), 1539. https://doi.org/10.3389/fpsyg.2019.01539

[2] Theo Araujo. 2018. Living up to the chatbot hype: The influence of anthropomorphic design cues and communicative agency framing on conversational agent and company perceptions. Computers in Human Behavior 85 (2018), 183-189. https://doi.org/10.1016/j.chb.2018.03.051

[3] Theo Araujo. 2020. Conversational Agent Research Toolkit: An alternative for creating and managing chatbots for experimental research. Computational Communication Research 2, 1 (2020), 35-51. https://doi.org/10.5117/CCR2020.1. 002.ARAU

[4] Christoph Bartneck, Takayuki Kanda, Omar Mubin, and Abdullah Al Mahmud 2009. Does the Design of a Robot Influence Its Animacy and Perceived Intelligence? International fournal of Social Robotics 1, 2 (2009), 195-204. https: //doi.org/10.1007/s12369-009-0013-7

[5] Christoph Bartneck, Dana Kulić, Elizabeth Croft, and Susana Zoghbi. 2009. Measurement Instruments for the Anthropomorphism, Animacy, Likeability, Per ceived Intelligence, and Perceived Safety of Robots. International fournal of Social Robotics 1, 1 (2009), 71-81. https://doi.org/10.1007/s12369-008-0001-3

[6] Pascal Boyer. 1996. What Makes Anthropomorphism Natural: Intuitive Ontology and Cultural Representations. The fournal of the Royal Anthropological Institute 2, 1 (1996), 83-97. https://doi.org/10.2307/3034634

[7] Elizabeth Broadbent. 2017. Interactions With Robots: The Truths We Reveal About Ourselves. Annual Review of Psychology 68, 1 (2017), 627-652. https: //doi.org/10.1146/annurev-psych-010416-043958

[8] John R Carlson and Robert W Zmud. 1999. Channel Expansion Theory and the Experiential Nature of Media Richness Perceptions. The Academy of Management fournal 42, 2 (1999), 153-170. https://doi.org/10.2307/257090

[9] Emily S Cross, Ruud Hortensius, and Agnieszka Wykowska. 2019. From social brains to social robots: applying neurocognitive insights to human-robot interaction. Philosophical Transactions of the Royal Society B: Biological Sciences 374 1771 (2019), 20180024. https://doi.org/10.1098/rstb.2018.0024

[10] Emily S Cross, Richard Ramsey, Roman Liepelt, Wolfgang Prinz, and Antonia de C Hamilton F. 2016. The shaping of social perception by stimulus and knowledge cues to human animacy. Philosophical transactions of the Royal Society of London.Series B, Biological sciences 371, 1686 (2016), 20150075. https://doi.org/10.1098/rstb.2015.0075

[11] Charles R Crowell, Jason C Deska, Michael Villano, Julaine Zenk, and John T. Roddy. 2019. Anthropomorphism of robots: Study of appearance and agency. fournal of Medical Internet Research 21, 5 (2019), e12629. https://doi.org/10.2196/ 12629

[12] Richard L Daft and Robert H Lengel. 1986. Organizational Information Requirements, Media Richness and Structural Design. Management Science 32, 5 (1986), 554-571. https://doi.org/10.1287/mnsc.32.5.554

[13] Luisa Damiano and Paul Dumouchel. 2018. Anthropomorphism in Human-Robot Co-evolution. Frontiers in Psychology 9 (2018), 468. https://doi.org/10.3389/fpsyg. 2018.00468

[14] E N Dunkin. 1981. Intelligence and Perception. In Psychology for Physiotherapists. Macmillan Education UK, London, 101-117. https://doi.org/10.1007/978-1-34916600-8_5

[15] Nicholas Epley and Adam Waytz. 2010. Mind Perception. John Wiley and Sons Ltd. https://doi.org/10.1002/9780470561119.socpsy001014

[16] Nicholas Epley, Adam Waytz, and John T Cacioppo. 2007. On seeing human: A three-factor theory of anthropomorphism. , 864-886 pages. https://doi.org/10. 1037/0033-295X.114.4.864

[17] Julia Fink. 2012. Anthropomorphism and Human Likeness in the Design of Robots and Human-Robot Interaction. In Social Robotics, Shuzhi Sam Ge, Oussama Khatib, John-John Cabibihan, Reid Simmons, and Mary-Anne Williams (Eds.). Springer Berlin Heidelberg, Berlin, Heidelberg, 199-208. https://doi.org/10.1007/978-3642-34103-8\{_\}20

[18] Asbjørn Følstad and Marita Skjuve. 2019. Chatbots for Customer Service: User Experience and Motivation. In Proceedings of the 1st International Conference on Conversational User Interfaces (CUI '19). Association for Computing Machinery, New York, NY, USA. https://doi.org/10.1145/3342775.3342784

[19] Asbjørn Følstad and Cameron Taylor. 2020. Conversational Repair in Chatbots for Customer Service: The Effect of Expressing Uncertainty and Suggesting Alternatives. In Chatbot Research and Design, Asbjørn Følstad, Theo Araujo, Symeon Papadopoulos, Effie Lai-Chong Law, Ole-Christoffer Granmo, Ewa Luger, and Petter Bae Brandtzaeg (Eds.). Springer International Publishing, Cham, 201214. https://doi.org/10.1007/978-3-030-39540-7\{ \}14

[20] Laure Gardelle and Sandrine Sorlin. 2018. Introduction: Anthropocentrism, egocentrism and the notion of Animacy Hierarchy. International fournal of Language and Culture 5, 2 (2018), 133-162. https://doi.org/10.1075/ijolc.00004.gar

[21] Eun Go and S Shyam Sundar. 2019. Humanizing chatbots: The effects of visual, identity and conversational cues on humanness perceptions. Computers in Human Behavior 97 (2019), 304-316. https://doi.org/10.1016/j.chb.2019.01.020
[22] Kurt Gray, Liane Young, and Adam Waytz. 2012. Mind Perception Is the Essence of Morality. Psychological Inquiry 23, 2 (2012), 101-124. https://doi.org/10.1080/ 1047840X.2012.651387

[23] David Griol, Javier Carbó, and José M Molina. 2013. AN AUTOMATIC DIALOG SIMULATION TECHNIQUE TO DEVELOP AND EVALUATE INTERACTIVE CONVERSATIONAL AGENTS. Applied Artificial Intelligence 27, 9 (2013), 759780. https://doi.org/10.1080/08839514.2013.835230

[24] Andrew F Hayes. 2018. Partial, conditional, and moderated moderated mediation: Quantification, inference, and interpretation. Communication Monographs 85, 1 (2018), 4-40. https://doi.org/10.1080/03637751.2017.1352100

[25] A F Hays. 2018. Introduction to Mediation, Moderation, and Conditional Process Analysis: A Regession Approach. Number August. Guilford publications. 3-4 pages.

[26] Anna Henschel, Guy Laban, and Emily S Cross. 2021. What Makes a Robot Social? A Review of Social Robots from Science Fiction to a Home or Hospital Near You. Current Robotics Reports (2021). https://doi.org/10.1007/s43154-020-00035-0

[27] Ruud Hortensius and Emily S Cross. 2018. From automata to animate beings: the scope and limits of attributing socialness to artificial agents. Annals of the New York Academy of Sciences 1426, 1 (2018), 93-110. https://doi.org/10.1111/nyas. 13727

[28] Ruud Hortensius, Felix Hekele, and Emily S Cross. 2018. The Perception of Emotion in Artificial Agents. IEEE Transactions on Cognitive and Developmental Systems 10, 4 (2018), 852-864. https://doi.org/10.1109/TCDS.2018.2826921

[29] Daniel Johnson and John Gardner. 2009. Exploring mindlessness as an explanation for the media equation: a study of stereotyping in computer tutorials. Personal and Ubiquitous Computing 13, 2 (2009), 151-163. https://doi.org/10.1007/s00779007-0193-9

[30] Guy Laban and Theo Araujo. 2020. The Effect of Personalization Techniques in Users' Perceptions of Conversational Recommender System. In IVA '20: Proceedings of the 20th ACM International Conference on Intelligent Virtual Agents (IVA '20). ACM, Virtual Event, Glasgow, Scotland UK, 3. https://doi.org/10.1145/ 3383652.3423890

[31] Guy Laban and Theo Araujo. 2020. Working Together with Conversational Agents: The Relationship of Perceived Cooperation with Service Performance Evaluations. In Lecture Notes in Computer Science (including subseries Lecture Notes in Artificial Intelligence and Lecture Notes in Bioinformatics), Følstad Asbjørn, Theo Araujo, Symeon Papadopoulos, Effie L Law, Ole-Christoffer Granmo, Ewa Luger, and Petter B Brandtzaeg (Eds.). Springer International Publishing, Cham, 215-228. https://doi.org/10.1007/978-3-030-39540-7\{_\}15

[32] Guy Laban, Jean-Noël George, Val Morrison, and Emily S. Cross. 2021. Tell me more! Assessing interactions with social robots from speech. Paladyn, Journal of Behavioral Robotics 12, 1 (2021), 136-159. https://doi.org/10.1515/pjbr-2021-0011

[33] Guy Laban, Val Morrison, and Emily S Cross. 2020. Let's Talk About It! Subjective and Objective Disclosures to Social Robots. In Companion of the 2020 ACM/IEEE International Conference on Human-Robot Interaction. Association for Computing Machinery, Cambridge, United Kingdom, 328-330. https: //doi.org/10.1145/3371382.3378252

[34] Antonio Lieto, Christian Lebiere, and Alessandro Oltramari. 2018. The knowledge level in cognitive architectures: Current limitations and possible developments. Cognitive Systems Research 48 (2018), 39-55. https://doi.org/10.1016/j.cogsys. 2017.05.001

[35] Phil McAleer, Barbara Mazzarino, Gaultiero Volpe, Antonio Camurri, Helena Patterson, and Frank Pollick. 2004. Perceiving Animacy and Arousal in Transformed Displays of Human Interaction. Journal of Vision 4, 8 (8 2004), 230. https://doi.org/10.1167/4.8.230

[36] Phil McAleer, Helena M Paterson, Barbara Mazzarino, and Frank E Pollick. 2005. Towards canonical views of animacy from scenes of human action. Fournal of Vision 5, 8 (9 2005), 936. https://doi.org/10.1167/5.8.936

[37] Barbara C N Müller, Xin Gao, Sari R R Nijssen, and Tom G E Damen. 2020. I, Robot: How Human Appearance and Mind Attribution Relate to the Perceived Danger of Robots. International fournal of Social Robotics (2020). https://doi.org/ 10.1007/s12369-020-00663-8

[38] Clifford Nass and Youngme Moon. 2000. Machines and Mindlessness: Social Responses to Computers. Fournal of Social Issues 56, 1 (2000), 81-103. https: //doi.org/10.1111/0022-4537.00153

[39] Clifford Nass, Jonathan Steuer, and Ellen R Tauber. 1994. Computers Are Social Actors. In Proceedings of the SIGCHI Conference on Human Factors in Computing Systems (CHI '94). Association for Computing Machinery, New York, NY, USA, 72-78. https://doi.org/10.1145/191666.191703

[40] Ulric Neisser, Gwyneth Boodoo, Thomas J Bouchard Jr., A Wade Boykin, Nathan Brody, Stephen J Ceci, Diane F Halpern, John C Loehlin, Robert Perloff, Robert J Sternberg, and Susana Urbina. 1996. Intelligence: Knowns and unknowns. American Psychologist 51, 2 (1996), 77-101. https://doi.org/10.1037/0003-066X.51.2.77

[41] Kristopher J Preacher and Andrew F Hayes. 2004. SPSS and SAS procedures for estimating indirect effects in simple mediation models. Behavior Research Methods, Instruments, \& Computers 36, 4 (2004), 717-731. https://doi.org/10.3758/ BF03206553 
[42] Byron Reeves and Clifford Ivar Nass. 1996. The media equation: How people treat computers, television, and new media like real people and places. Cambridge university press.

[43] Giulio Sandini, Vishwanathan Mohan, Alessandra Sciutti, and Pietro Morasso 2018. Social Cognition for Human-Robot Symbiosis - Challenges and Building Blocks. Frontiers in Neurorobotics 12 (2018), 34. https://doi.org/10.3389/fnbot. 2018.00034

[44] Brian J Scholl and Patrice D Tremoulet. 2000. Perceptual causality and animacy. Trends in Cognitive Sciences 4, 8 (8 2000), 299-309. https://doi.org/10.1016/S13646613(00)01506-0

[45] Bayan Abu Shawar and Eric Atwell. 2007. Chatbots: are they really useful? $L d v$ Forum 22, 1 (2007), 29-49.

[46] Marita Skjuve, Ida Haugstveit, Asbjørn Følstad, and Petter Brandtzaeg. 2019 Help! Is my chatbot falling into the uncanny valley? An empirical study of user experience in human-chatbot interaction. Human Technology 15 (2019), 30-54 https://doi.org/10.17011/ht/urn.201902201607

[47] Robert J Sternberg. 1999. The Theory of Successful Intelligence. Review of General Psychology 3, 4 (12 1999), 292-316. https://doi.org/10.1037/1089-2680.3.4.292

[48] Robert J Sternberg. 2003. A Broad View of Intelligence: The Theory of Successful Intelligence. Consulting Psychology fournal: Practice and Research 55, 3 (2003),
139-154. https://doi.org/10.1037/1061-4087.55.3.139

[49] S S Sundar and Sampada S Marathe. 2010. Personalization versus Customization: The Importance of Agency, Privacy, and Power Usage. Human Communication Research 36, 3 (2010), 298-322. https://doi.org/10.1111/j.1468-2958.2010.01377.x

[50] Michelle M E Van Pinxteren, Mark Pluymaekers, and Jos G A M Lemmink. 2020. Human-like communication in conversational agents: a literature review and research agenda. Fournal of Service Management (2020). https://doi.org/10.1108/ JOSM-06-2019-0175

[51] Hilde A M Voorveld and Theo Araujo. 2020. How Social Cues in Virtual Assistants Influence Concerns and Persuasion: The Role of Voice and a Human Name. Cyberpsychology, Behavior, and Social Networking (2020). https://doi.org/10.1089/ cyber.2019.0205

[52] Rebecca M Warner and David B Sugarman. 1986. Attributions of personality based on physical appearance, speech, and handwriting. Fournal of Personality and Social Psychology 50, 4 (1986), 792-799. https://doi.org/10.1037/0022-3514.50.4.792

[53] Adam Waytz, John Cacioppo, and Nicholas Epley. 2010. Who Sees Human?: The Stability and Importance of Individual Differences in Anthropomorphism. Perspect Psychol Sci 5, 3 (2010), 219-232. https://doi.org/10.1177/1745691610369336 